\title{
Erratum: Dipole selection rules for optical transitions in the fec and bec lattices
}

[Phys. Rev. B 21, $5572(1980)]$

W. Eberhardt and F. J. Himpsel

The caption of Table IV contains two errors: “( $(0)$ is for $\vec{A}$ normal $\Sigma$; parallel $x$ " should read " $(0)$ is for $\vec{A}$ normal $\Sigma$; parallel $z$ "; “ $(X)$ is for $\vec{A}$ normal $\Sigma$; parallel $y$ " should read " $(X)$ is for $\vec{A}$ normal $\Sigma$; parallel $x-y$ ".

Table VI should be replaced by the following table.

TABLE VI. Allowed dipole transitions at $N .(+)$ is for $\vec{A}$ parallel $x+y ; \vec{A} \cdot \vec{p}$ is represented by $N_{1^{\prime}}$. (0) is for $\vec{A}$ parallel $z ; \overrightarrow{\mathrm{A}} \cdot \overrightarrow{\mathrm{p}}$ is represented by $N_{3^{\prime}}$. (X) is for $\overrightarrow{\mathrm{A}}$ parallel $x-y ; \overrightarrow{\mathrm{A}} \cdot \overrightarrow{\mathrm{p}}$ is represented by $N_{4^{\prime}}$.

\begin{tabular}{|c|c|c|c|c|c|c|c|c|}
\hline$D_{2 h}$ & $N_{1}$ & $N_{2}$ & $N_{3}$ & $N_{4}$ & $N_{1^{\prime}}$ & $N_{2}^{\prime}$ & $N_{3^{\prime}}$ & $N_{4^{\prime}}$ \\
\hline$N_{1}$ & $\cdots$ & $\cdots$ & $\cdots$ & $\cdots$ & + & $\cdots$ & 0 & $X$ \\
\hline$N_{2}$ & $\cdots$ & $\cdots$ & $\cdots$ & $\cdots$ & $\cdots$ & + & $X$ & 0 \\
\hline $\mathrm{N}_{3}$ & $\cdots$ & $\cdots$ & $\cdots$ & $\cdots$ & 0 & $X$ & + & $\cdots$ \\
\hline$N_{4}$ & $\cdots$ & $\cdots$ & $\ddot{*}$ & $\ddot{*}$ & $X$ & 0 & $\cdots$ & + \\
\hline$N_{1^{\prime}}$ & + & $\cdots$ & 0 & $X$ & $\cdots$ & $\cdots$ & $\cdots$ & $\cdots$ \\
\hline$N_{2^{\prime}}$ & $\because \cdot$ & + & $X$ & 0 & $\cdots$ & $\cdots$ & $\cdots$ & $\cdots$ \\
\hline$N_{3^{\prime}}$ & 0 & $X$ & + & $\cdots$ & $\cdots$ & $\cdots$ & $\cdots$ & $\cdots$ \\
\hline$N_{4^{\prime}}$ & $X$ & 0 & $\cdots$ & + & $\cdots$ & $\cdots$ & $\cdots$ & $\cdots$ \\
\hline
\end{tabular}

\section{Erratum: Active nonlinear spectroscopy of biexcitons in semiconductors: Propagation effects and Fano interferences \\ [Phys. Rev. B 23, 841 (1981)]}

A. Maruani and D. S. Chemia

An error has unfortunately escaped our attention in the text of our article. On page 852 of the text two signs are incorrect, giving $\nu=-q^{-1}\left[\hbar \omega=\frac{1}{2}\left(W_{x_{2}}+\Gamma_{x_{2}} / q\right)\right]$.

\section{Erratum: Extension of liquid-metal theory to dense partially ionized plasmas} [Phys. Rev. B 21, 3140 (1980)]

Marvin Ross

The term $U_{\text {ei }}$ appears twice in Eq. (10). The first one should be omitted. The term $N_{\text {core }}$ in Eqs. (37) and (38) should be replaced by $N_{\text {cond }}$. 